\title{
ESPECTRO DE FUCIK PARA UN PROBLEMA DE VALOR FRONTERA DE TERCER ORDEN
}

\section{Santiago Rojas ${ }^{*}$}

Resumen: En este trabajo se estudia el Espectro de Fucik para el problema de valor frontera

$$
\left\{\begin{array}{l}
y^{\prime \prime \prime}+\mu y^{+}-\nu y^{-}=0 \quad \text { en }\langle 0,1\rangle \\
y(0)=y^{\prime}(0)=y(1)=0
\end{array}\right.
$$

y se describen las curvas de Fucik contenidas en dicho espectro.

Palabras clave: Espectro de Fucik, Curvas de Fucik, ecuación de tercer orden.

\section{FUCIK SPECTRUM FOR A THIRD ORDER BOUNDARY VALUE PROBLEM}

Abstract: In this work, the Fucik Spectrum for the boundary value problem

$$
\left\{\begin{array}{l}
y^{\prime \prime \prime}+\mu y^{+}-\nu y^{-}=0 \quad \text { on }\langle 0,1\rangle \\
y(0)=y^{\prime}(0)=y(1)=0
\end{array}\right.
$$

is studied, and the Fucik curves contained in this spectrum are described.

Key words: Fucik spectrum, Fucik Curves, Third order equation.

El Espectro de Fucik para el problema de valor frontera

$$
\left\{\begin{array}{l}
y^{\prime \prime \prime}+\mu y^{+}-\nu y^{-}=0 \quad \text { en }\langle 0,1\rangle \\
y(0)=y^{\prime}(0)=y(1)=0
\end{array}\right.
$$

donde $y^{+}=\max \{y, 0\}, y^{-}=\max \{-y, 0\}$, es el conjunto de puntos $(\mu, \nu) \in \mathbb{R}^{2}$ para los cuales el problema (1.1) tiene una solución no trivial. Para problemas de segundo orden, en [2] y [3] se calcula el espectro de Fucik para problemas con condiciones de frontera tipo Dirichlet, y en [4] se estudia el espectro de Fucik para el problema con condiciones de frontera Sturm-Liouville. En general, el cálculo del espectro de Fucik es difícil aún para los problemas con ecuaciones diferenciales ordinarias y poco se sabe sobre el espectro de Fucik para los problemas con ecuaciones diferenciales parciales. En [1] De Figueiredo y Gossez calculan la primera curva no trivial del espectro de Fucik para el problema

$$
\left\{\begin{aligned}
-\Delta u & =\mu u^{+}-\nu u^{-} \\
\left.u\right|_{\partial \Omega} & =0
\end{aligned}\right.
$$

donde $\Delta$ es el operador Laplaciano. Y para el caso de un operador autoadjunto sobre $L^{2}(\Omega)$ con resolvente compacto, en [5] se demuestra que a través de cada par $\left(\lambda_{k}, \lambda_{k}\right)$ pasan dos curvas de Fucik $\left(\lambda_{k}\right.$ son los autovalores del operador), las cuales en algunos casos pueden coincidir. En [1] y [5] se ha hecho el estudio en forma variacional, pero en nuestro caso (problema (1.1)) no es posible usar ese enfoque pues el problema lineal no es autoadjunto. Más bien, usaremos el hecho de que, haciendo $u(t)=\frac{y(t)}{ \pm y^{\prime \prime}(0)}$, las soluciones $y(t)$ de (1.1) pueden ser vistas como soluciones de los problemas de Cauchy

$$
\left\{\begin{aligned}
u^{\prime \prime \prime}+\mu u^{+} & -\nu u^{-}=0 \\
u(0)=u^{\prime}(0)=0, & u^{\prime \prime}(0)= \pm 1
\end{aligned}\right.
$$

\footnotetext{
*UNMSMS, Facultad de Ciencias Matemáticas, e-mail: srojasr@unmsm.edu.pe
} 
Así, el Espectro de Fucik de (1.1) estará formado por aquellos pares $(\mu, \nu)$ tales que 1 es un cero de las soluciones de (1.2). El método seguido en el trabajo se basa en un detallado estudio de los ceros de las soluciones de (1.2) y su dependencia de $\mu$ y $\nu$, lo cual desarrollamos en la sección 2. Finalmente, en la. sección 3 obtenemos una completa descripción del espectro de Fucik de (1.1).

\section{Resultados Preliminares}

En esta sección consideramos los resultados técnicos necesarios para nuestro estudio. Empezamos definiendo $y_{i}(t, \alpha), i=0,1,2$, como las soluciones de los problemas de Cauchy

$$
\begin{array}{ccc}
y_{i}^{\prime \prime \prime}+\alpha^{3} y_{i}=0, & \\
y_{0}(0, \alpha)=1, & y_{0}^{\prime}(0, \alpha)=0, & y_{0}^{\prime \prime}(0, \alpha)=0 \\
y_{1}(0, \alpha)=0, & y_{1}^{\prime}(0, \alpha)=1, & y_{1}^{\prime \prime}(0, \alpha)=0 \\
y_{2}(0, \alpha)=0, & y_{2}^{\prime}(0, \alpha)=0, & y_{2}^{\prime \prime}(0, \alpha)=1
\end{array}
$$

donde $\alpha>0$. Resolviendo, tenemos que

$$
y_{0}(t, \alpha)=z_{0}(\alpha t), \quad y_{1}(t, \alpha)=\frac{1}{\alpha} z_{1}(\alpha t), \quad y_{2}(t, \alpha)=\frac{1}{\alpha^{2}} z_{2}(\alpha t),
$$

con

$$
\begin{aligned}
& z_{0}(s)=\frac{1}{3}\left[e^{-s}+2 e^{s / 2} \cos \left(\frac{\sqrt{3}}{2} s\right)\right] \\
& z_{1}(s)=\frac{1}{3}\left[-e^{-s}+2 e^{s / 2} \sin \left(\frac{\sqrt{3}}{2} s+\frac{\pi}{6}\right)\right], \\
& z_{2}(s)=\frac{1}{3}\left[e^{-s}+2 e^{s / 2} \sin \left(\frac{\sqrt{3}}{2} s-\frac{\pi}{6}\right)\right]
\end{aligned}
$$

Observación 1.1. $z_{0}^{\prime}(t)=-z_{2}(t), \quad z_{1}^{\prime}(t)=z_{0}(t), \quad z_{2}^{\prime}(t)=z_{1}(t)$.

El siguiente Lema se demuestra usando cálculo diferencial.

Lema 1.1. Los ceros $\rho_{1}, \sigma_{1}$ y $\tau_{1}$ de las funciones $z_{0}, z_{1}$ y $z_{2}$ son simples y pueden ser ordenados como sigue

$$
0<\rho_{1}<\sigma_{1}<\tau_{1}<\cdots<\rho_{n}<\sigma_{n}<\tau_{n}<\cdots .
$$

Prueba. Haciendo un cálculo directo obtenemos que

$$
z_{0}\left(\frac{\pi}{\sqrt{3}}\right)>0 \quad y \quad z_{0}\left(\frac{2 \pi}{\sqrt{3}}\right)<0
$$

entonces existe $\tau_{1} \in\left\langle\frac{\pi}{\sqrt{3}}, \frac{2 \pi}{\sqrt{3}}\right\rangle$ tal que $z_{0}^{\prime}\left(\tau_{1}\right)=0$. De la observación (2.1), $\tau_{1}$ es cero de $z_{2}$. Como $z_{2}(0)=0$, por el Teorema de Rolle existe $\sigma_{1} \in\left\langle 0, \tau_{1}\right\rangle$ tal que $z_{2}^{\prime}\left(\sigma_{1}\right)=0$. De la observación (2.1), $\sigma_{1}$ es cero de $z_{1}$. Y como $z_{1}(0)=0$, nuevamente por el Teorema de Rolle existe $\rho_{1} \in\left\langle 0, \sigma_{1}\right\rangle$ tal que $z_{1}^{\prime}\left(\rho_{1}\right)=0$. Entonces $\rho_{1}$ es cero de $z_{0}$. Así tenemos

$$
0<\rho_{1}<\sigma_{1}<\tau_{1} .
$$

En general, el esquema es análogo, primero se tiene la existencia de $\tau_{i}, \forall i$ y por el Teorema de Rolle y la observación (2.1) tenemos la existencia de $\sigma_{i}$ y $\rho_{i}$. Con el mismo argumento se prueba que los ceros de $z_{i}$ y $z_{i+1}$ alternan $\left(z_{3}=z_{0}\right)$ y tenemos el resultado . 
A continuacion presentamos el resultado que muestra la existencia y una propiedad importante del primer cero de la solución del problema de Cauchy

$$
\left\{\begin{array}{c}
y^{\prime \prime \prime}+\alpha^{3} y=0, \\
y(0)=0, \quad y^{\prime}(0)=q, \quad y^{\prime \prime}(0)=r .
\end{array}\right.
$$

Proposición 1.1. Sean $\alpha>0, q \in \mathbb{R}$ y $r \neq 0$ tales que $s=q / r \geq 0$ y sea y la solución de (2.2). Entonces la función y tiene un primer cero positivo $\tau(s, \alpha)$ tal que

$$
y^{\prime}(\tau(s, \alpha)) y^{\prime \prime}(\tau(s, \alpha))>r q \geq 0 .
$$

Además, para $s>0, \tau(s, \alpha) \in\left\langle\frac{\sigma_{1}}{\alpha}, \frac{\tau_{1}}{\alpha}\right\rangle$.

Prueba. Resolviendo (2.2) tenemos que la solución es

$$
y(t)=\left(\frac{r}{3 \alpha^{2}}-\frac{q}{3 \alpha}\right) e^{-\alpha t}+e^{\frac{\alpha}{2} t}\left[\left(-\frac{r}{3 \alpha^{2}}+\frac{q}{3 \alpha}\right) \cos \frac{\sqrt{3}}{2} \alpha t+\left(\frac{r}{\alpha^{2} \sqrt{3}}+\frac{q}{\alpha \sqrt{3}}\right) \operatorname{sen} \frac{\sqrt{3}}{2} \alpha t\right]
$$

y reagrupando los términos llegamos a

$$
y(t)=\frac{r}{\alpha^{2}} z_{2}(\alpha t)+\frac{q}{\alpha} z_{1}(\alpha t),
$$

de donde

$$
y(t)=r\left[s \frac{z_{1}(\alpha t)}{\alpha}+\frac{z_{2}(\alpha t)}{\alpha^{2}}\right] .
$$

Como $z_{1}$ y $z_{2}$ son positivas en $\left\langle 0, \sigma_{1}\right]$, tenemos que

$$
y(t)>0 \quad, \quad \forall t \in\left\langle 0, \frac{\sigma_{1}}{\alpha}\right] .
$$

También, para $s>0$,

$$
y\left(\frac{\tau_{1}}{\alpha}\right)=r s \frac{z_{1}\left(\tau_{1}\right)}{\alpha}<0 .
$$

De ahí se tiene que existe un primer cero positivo $\tau(s, \alpha) \in\left\langle\frac{\sigma_{1}}{\alpha}, \frac{\tau_{1}}{\alpha}\right\rangle$. Ahora calculamos

$$
\left.0=-\alpha^{3} \int_{0}^{\tau} y y^{\prime} d t=\int_{0}^{\tau} y^{\prime \prime \prime} y^{\prime} d t=y^{\prime \prime} y^{\prime}\right]_{0}^{\tau}-\int_{0}^{\tau}\left(y^{\prime \prime}\right)^{2} d t
$$

donde $\tau=\tau(s, \alpha)$. Entonces

$$
y^{\prime \prime}(\tau(s, \alpha)) y^{\prime}(\tau(s, \alpha))-r q>0 .
$$

Observación 1.2. $\tau(s, \alpha)$ es un cero simple.

En efecto, no perdamos de vista que $z_{2}(\alpha t)$ es positivo en $\langle 0, \tau(s, \alpha)\rangle$, pero $z_{0}(\alpha t)$ y $z_{1}(\alpha t)$ son negativos. Luego

i) Si $q \geq 0, r>0$, entonces $y(t)>0$ en $\langle 0, \tau(s, \alpha)\rangle$. También

$$
\begin{gathered}
y^{\prime}(t)=r\left[s z_{1}^{\prime}(\alpha t)+\frac{1}{\alpha} z_{2}^{\prime}(\alpha t)\right]=r\left[s z_{0}(\alpha t)+\frac{1}{\alpha} z_{1}(\alpha t)\right]<0 \quad \mathrm{y} \\
y^{\prime \prime}(t)=r\left[s \alpha z_{0}^{\prime}(\alpha t)+z_{1}^{\prime}(\alpha t)\right]=r\left[-s \alpha z_{2}(\alpha t)+z_{0}(\alpha t)\right]<0 .
\end{gathered}
$$

ii) Si $q<0, r<0$, entonces $y(t)<0$ en $\langle 0, \tau(s, \alpha)\rangle$. También

$$
\begin{gathered}
y^{\prime}(t)=r\left[s z_{0}(\alpha t)+\frac{1}{\alpha} z_{1}(\alpha t)\right]>0 \quad \mathrm{y} \\
y^{\prime \prime}(t)=r\left[-s \alpha z_{2}(\alpha t)+z_{0}(\alpha t)\right]>0 .
\end{gathered}
$$




\section{El Espectro de Fucik}

En esta sección, estudiamos el Espectro de Fucik para el problema de valor frontera

$$
\left\{\begin{array}{r}
y^{\prime \prime \prime}+\mu y^{+}-\nu y^{-}=0 \\
y(0)=y^{\prime}(0)=y(1)=0 .
\end{array}\right.
$$

El Espectro de Fucik $F$ de (3.1) es el conjunto de pares $(\mu, \nu) \in \mathbb{R}^{2}$ para los cuales el problema (3.1) tiene solución no trivial.

Empezamos estudiando los autovalores del problema

$$
\left\{\begin{array}{c}
y^{\prime \prime \prime}+\lambda y=0 \\
y(0)=y^{\prime}(0)=y(1)=0 .
\end{array}\right.
$$

Proposición 2.1. Los autovalores $\lambda_{i}$ del problema (3.2) son simples y tales que

$$
0<\lambda_{1}<\lambda_{2}<\cdots<\lambda_{n}<\cdots .
$$

Prueba. Sea $\lambda$ un autovalor de (3.2) y su correspondiente autofunción $y$. Haciendo cálculos obtenemos

$$
\begin{aligned}
0 & =\int_{0}^{1} y^{\prime \prime \prime} y d t+\lambda \int_{0}^{1} y^{2} d t \\
& \left.=\left(y^{\prime \prime} y-\frac{1}{2} y^{\prime 2}\right)\right]_{0}^{1}+\lambda \int_{0}^{1} y^{2} d t \\
& =-\frac{1}{2} y^{\prime 2}(1)+\lambda \int_{0}^{1} y^{2} d t,
\end{aligned}
$$

lo cual prueba que $\lambda>0$. Y como $y^{\prime \prime}(0) \neq 0$, tenemos que $u=\frac{y}{y^{\prime \prime}(0)}$ satisface el problema de Cauchy

$$
\left\{\begin{array}{c}
u^{\prime \prime \prime}+\lambda u=0 \\
u(0)=u^{\prime}(0)=0, \quad u^{\prime \prime}(0)=1
\end{array}\right.
$$

De (2.1), $u(t)=\frac{1}{\lambda^{2 / 3}} z_{2}\left(\lambda^{1 / 3} t\right)$. Y por la Proposición (2.1), $\lambda$ es simple. Así, los autovalores $\lambda_{i}$ pueden ser calculados a partir de los ceros de $z_{2}$.

Proposición 2.2. El $i$-ésimo autovalor de (3.2) es $\lambda_{i}=\tau_{i}^{3}$, donde $\lambda_{i}$ es el i-ésimo cero de $z_{2}\left(\lambda_{i} t\right)$.

Prueba. Derivando y usando la Observación 2.1, tenemos

$$
\begin{aligned}
z_{2}^{\prime}\left(\tau_{i} t\right) & =\tau_{i} z_{1}\left(\tau_{i} t\right) \\
z_{2}^{\prime \prime}\left(\tau_{i} t\right) & =\tau_{i}^{2} z_{0}\left(\tau_{i} t\right) \\
z_{2}^{\prime \prime \prime}\left(\tau_{i} t\right) & =-\tau_{i}^{3} z_{2}\left(\tau_{i} t\right) .
\end{aligned}
$$

Asimismo, $z_{2}(0)=z_{2}^{\prime}(0)=0$ y $z_{2}(1)=0$, con lo cual se verifica que $z_{2}\left(\tau_{i} t\right)$ es una autofunción de (3.1) correspondiente al autovalor $\lambda_{i}=\tau_{i}^{3}$.

Ahora demostramos que el espectro de Fucik $F$ de (3.1) es no vacío.

Proposición 2.3. $F \neq \emptyset$ 
Prueba. Se sabe que para cualquier función $y$ se verifica la igualdad $y=y^{+}-y^{-}$. Entonces

$$
\left\{\begin{array}{r}
z_{2}^{\prime \prime \prime}+\lambda_{i} z_{2}^{+}-\lambda_{i} z_{2}^{-}=0 \\
z_{2}(0)=z_{2}^{\prime}(0)=z_{2}(1)=0,
\end{array}\right.
$$

donde $\lambda_{i}=\tau_{i}^{3}$. Por tanto

$$
\forall i \geq 1, \quad\left(\lambda_{i}, \lambda_{i}\right) \in F .
$$

El siguiente resultado muestra que el Espectro de Fucik $F$ es simétrico respecto a la recta $\mu=\nu$.

Proposición 2.4. $(\mu, \nu) \in F$ si y sólo si $(\nu, \mu) \in F$.

Prueba. Sea $(\mu, \nu) \in F$. Entonces existe $u \neq 0$ tal que

$$
\left\{\begin{aligned}
u^{\prime \prime \prime}+\mu u^{+}-\nu u^{-} & =0 \\
u(0)=u^{\prime}(0)=u(1) & =0 .
\end{aligned}\right.
$$

Luego, como $(-u)^{+}=u^{-}$y $(-u)^{-}=u^{+}$, tenemos que

$$
\left\{\begin{array}{r}
(-u)^{\prime \prime \prime}+\nu(-u)^{+}-\mu(-u)^{-}=0 \\
-u(0)=-u^{\prime}(0)=-u(1)=0 .
\end{array}\right.
$$

Por tanto, $(\nu, \mu) \in F$. El recíproco es análogo.

Ahora presentamos el principal resultado del trabajo, el cual brinda una descripción completa del espectro de Fucik de (3.1).

\section{Proposición 2.5.}

$$
F=\bigcup_{i=1}^{\infty}\left(F_{i}^{+} \cup F_{i}^{-}\right)
$$

donde

$$
\begin{aligned}
& F_{1}^{+}=\left\{\left(\lambda_{1}, \nu\right) / \nu \in \mathbb{R}\right\}, \\
& F_{1}^{-}=\left\{\left(\mu, \lambda_{1}\right) / \mu \in \mathbb{R}\right\} \\
& F_{i}^{+}=\left\{(\mu, \nu) / \mu=\alpha^{3}, \nu=\beta^{3}, \phi_{i}^{+}(\alpha, \beta)=1\right\}, i>1, \\
& F_{i}^{-}=\left\{(\mu, \nu) / \mu=\alpha^{3}, \nu=\beta^{3}, \phi_{i}^{-}(\alpha, \beta)=1\right\}, i>1,
\end{aligned}
$$

con $\lambda_{1}$ el primer autovalor de (3.2), $\phi_{i}^{+}(\alpha, \beta)$ el i-ésimo cero positivo de la solución de

$$
\left\{\begin{aligned}
y^{\prime \prime \prime}+\alpha^{3} y^{+}-\beta^{3} y^{-} & =0 \\
y(0)=y^{\prime}(0)=0, \quad y^{\prime \prime}(0) & =1
\end{aligned}\right.
$$

y $\phi_{i}^{-}(\alpha, \beta)$ el i-ésimo cero positivo de la solución de

$$
\left\{\begin{aligned}
y^{\prime \prime \prime}+\alpha^{3} y^{+}-\beta^{3} y^{-} & =0 \\
y(0)=y^{\prime}(0)=0, \quad y^{\prime \prime}(0) & =-1 .
\end{aligned}\right.
$$


Prueba. En primer lugar, notamos que para toda solución $y$ de $(3.1)$, las funciones $u_{1}=\frac{y}{y^{\prime \prime}(0)}$ y $u_{2}=\frac{y}{-y^{\prime \prime}(0)}$ son soluciones de los problemas (3.3) y (3.4), respectivamente. Por ello, el espectro de Fucik de (3.1) está formado por aquellos pares $(\mu, \nu)$ tales que 1 es un cero de las soluciones de (3.3) y (3.4). Ahora, como $\lambda_{1}=\tau_{1}^{3}\left(\tau_{1}\right.$ es el primer cero positivo de $\left.z_{2}\left(\tau_{1} t\right)\right)$, tenemos que

$$
\phi_{1}^{+}(\alpha, \beta)=\tau_{1} \quad y \quad z_{2}\left(\tau_{1} t\right)>0, \quad \forall t \in\langle 0,1\rangle .
$$

Entonces $z_{2}^{+}=z_{2} \quad$ y $\quad z_{2}^{-}=0$. Luego

$$
z_{2}^{\prime \prime \prime}\left(\tau_{1} t\right)+\lambda_{1} z_{2}^{+}\left(\tau_{1} t\right)-\nu z_{2}^{-}\left(\tau_{1} t\right)=0, \quad \forall \nu \in \mathbb{R} .
$$

De ahí que $\left(\lambda_{1}, \nu\right) \in F, \forall \nu \in \mathbb{R}$. Y por simetría, también $\left(\mu, \lambda_{1}\right) \in F, \forall \nu \in \mathbb{R}$.

De la Proposición 2.1 se obtiene que $\phi_{i}^{+}(\alpha, \beta), i>1$, existe sólo para $\alpha>0$ y $\beta>0$. Y por simetría, también $\phi_{i}^{-}(\alpha, \beta), i>1$, existe sólo para $\alpha>0$ y $\beta>0$.

Por tanto,

$$
\begin{array}{rlrl}
(\mu, \nu) \in F \Leftrightarrow \quad & (\mu, \nu) & =\left(\lambda_{1}, \nu\right), & \forall \nu \in \mathbb{R} \\
\text { ó } \quad(\mu, \nu) & =\left(\mu, \lambda_{1}\right), \quad \forall \mu \in \mathbb{R} \\
\text { ó } \quad(\mu, \nu) & =\left(\alpha^{3}, \beta^{3}\right) \wedge \phi_{i}^{+}(\alpha, \beta)=1, \quad i>1 \\
\text { ó } \quad(\mu, \nu) & =\left(\alpha^{3}, \beta^{3}\right) \wedge \phi_{i}^{-}(\alpha, \beta)=1, \quad i>1,
\end{array}
$$

con lo cual hemos demostrado el resultado. 


\section{Bibliografía}

[1] Figueiredo, D. G. and Gossez, J. P. (1994).On the first curve of the Fucik Spectrum of an elliptic operator. Differential Integral Equations 7, 1285-1302.

[2] Fucik, S. and Kufner A. (1980).Nonlinear Differential Equations. Elsevier Scientific Publishing Company. The Netherlands.

[3] Rojas, S. (2002). Un ejemplo del Espectro de Fucik. PESQUIMAT Vol V, No. 2 : 55-63.

[4] Rojas, S. (2006). Espectro de Fucik para el problema de valor frontera Sturm-Liouville. PESQUIMAT Vol IX, No. $2: 31-49$.

[5] Schechter, M. (1994). The Fucik Spectrum. Indiana University Mathematics Journal, Vol. 43 No. 4 : 1139-1157. 